\title{
Relevance of Heparan Sulfate and Heparanase to Severity of COVID-19 in the Elderly
}

\author{
Yona Nadir, MD, PhD ${ }^{1,2}$ Benjamin Brenner, $\mathrm{MD}^{1,2}$ \\ ${ }^{1}$ Thrombosis and Hemostasis Unit, Rambam Health Care Campus, \\ Haifa, Israel \\ 2 The Ruth and Bruce Rappaport Faculty of Medicine, Technion, \\ Haifa, Israel
}

Semin Thromb Hemost 2021;47:348-350.

The prevalence of arterial and venous thrombosis markedly rises with age. The annual rate of venous thromboembolism (VTE) is estimated at $1 / 100$ patients above 80 years of age. ${ }^{1}$ While aging is associated with some changes in the levels of coagulation proteins ${ }^{2}$ and activation markers, ${ }^{3}$ this does not explain the exponential increase in VTE risk observed in octogenarians.

Accordingly, such findings may be attributed to several factors. It has been recently demonstrated that coagulant activity under flow is mainly controlled by residual tissue factor (TF)/collagen and endothelial glycocalyx. ${ }^{4}$ Given that the vessel wall is a major player in hemostasis, endothelial dysfunction, which increases with age, likely contributes to the development of thrombosis in cardiovascular diseases. Rapidly growing evidence links particulate air pollution exposure with an increased risk of respiratory and cardiovascular disease. ${ }^{5}$ In the coronavirus disease 2019 (COVID-19) pandemic, the elderly are particularly vulnerable to severe vascular complications, including deep vein thrombosis and pulmonary embolism. This population appears to be particularly exposed in certain densely populated and air-polluted urban areas.

\section{The Immune Response in the Elderly}

Similar to the COVID-19 infection, the majority of deaths associated with influenza virus infections occur in individuals over 65 years of age in industrialized countries. ${ }^{6}$ Both the adaptive immune system and the innate response are altered during viral infections in the elderly. ${ }^{7,8}$ Various age-related defects have been found in the $\mathrm{T}$ cell compartment. ${ }^{9,10}$ Humoral responses are also affected, with attenuated titers of antibodies and abnormalities in the proportions of naive and memory B cell subsets. ${ }^{11,12}$ Experimental studies ${ }^{13}$ have demonstrated that dendritic cells (DCs), which are the main antigen-presenting cells

published online January 11, 2021
Issue Theme Maintaining Hemostasis and Preventing Thrombosis in COVID-19 -Part II; Guest Editors: Emmanuel J. Favaloro, PhD, FFSc (RCPA) and Giuseppe Lippi, MD.
Address for correspondence Yona Nadir, MD, PhD, Thrombosis and Hemostasis Unit, Rambam Health Care Campus, Haifa 3109601, Israel (e-mail: y_nadir@rambam.health.gov.il).

(APCs), from aged individuals have a reduced antigencapture capacity, impaired migration, and diminished $\mathrm{T}$ cell activation ability. Additionally, an increase in basal levels of various inflammatory cytokines (e.g., macrophage inflammatory protein 1 , tumor necrosis factor $\alpha$, gamma interferon, interleukin-1 [IL-1], IL-6), has consistently been described in the elderly. ${ }^{14,15}$ Elevated cytokine levels may contribute to enhanced lung tissue damage and increased mortality related to infections in elderly population.

\section{Heparanase Enhances the Immune Cell Activity}

Heparanase is a proinflammatory and proangiogenic protein, capable of degrading heparan sulfate (HS) chains both at cell surface and in the extracellular matrix. Several lines of evidence point to involvement of heparanase in the enhancement of innate and adaptive immune responses. Heparanase is found to be required for activation and function of macrophages, which is reported to be attributed to a linear cascade by which heparanase activates Erk, p38, and JNK signaling in macrophages, leading to induction of cytokine expression. ${ }^{16}$ Overexpression of heparanase enhances $T$ lymphocyte activity and intensifies the inflammatory response in a murine model of collagen-induced rheumatoid arthritis. ${ }^{17}$ Notably, transgenic mice overexpressing human heparanase (Hpa-tg) displayed an earlier response and developed more severe symptoms. Examination of cells from thymus, spleen, and lymph nodes demonstrated increased proportions of macrophages, APCs, plasmacytoid DCs, and CD4+ and CD8+ T cells, while splenic lymphocytes from Hpa-tg mice exhibited higher proliferation activity. These results suggest that augmented expression of heparanase can boost both the innate and adaptive immune system and propagate inflammatory reactions, ${ }^{17}$ which could be relevant to severe presentations of COVID-19. (c) 2021. Thieme. All rights reserved. Thieme Medical Publishers, Inc., 333 Seventh Avenue, 18th Floor, New York, NY 10001, USA
DOI https://doi.org/ 10.1055/s-0040-1722293. ISSN 0094-6176. 


\section{Heparanase, Heparan Sulfate and Aging}

Age-associated changes in glycosaminoglycans (GAGs) have been explored in several body organs. Levels of GAGs in lung specimens from different age groups were found to be lower in the elderly group (over 70 years of age) compared with younger groups (30-49 years of age). ${ }^{18}$ Hence, lung senescence has been associated with uniform decrease of all the GAG types in the lung. It was previously shown that HS content decreased during skin aging. Degradation of HS was reported to result in marked reduction in binding activity of the basement membrane for vascular endothelial growth factor, fibroblast growth factors 2 and 7 at the dermalepidermal junction. ${ }^{19}$ HS degradation was observed not only in acutely ultraviolet B (UV-B) irradiated skin, but also in skin chronically exposed to sun. These findings suggest that chronic UV-B exposure may facilitate photoaging via heparanase activation. ${ }^{19}$

A considerable amount of evidence supports the involvement of GAGs and heparanase in vascular aging. Nitschmann et al evaluating age-associated differences in arterial wall structure and function of prepubertal and adult rabbits, revealed significantly increased HS and chondroitin sulfate content in both intima and media of aorta, in rabbit pups. ${ }^{20}$ Likewise, rabbit pups demonstrated significant elevation in antithrombin activity related to HS. These findings suggest that arteries in young animals may have greater antithrombotic potential, which could be partly related to increased HS. Another study from the same group, focusing on venous system, assessed the inferior vena cava (IVC) wall morphology, GAG mass, and GAG antithrombin activity. The demonstrated differences in antithrombotic properties of IVC walls in rabbit pups and adults could contribute to the reduced risk of VTE in the young. ${ }^{21}$

Increased expression of tumor suppressor protein 53 (p53) is implicated in vascular senescence. ${ }^{22}$ Recently, Bochenek et al reported elevated endothelial heparanase expression associated with doxorubicin-induced p53 overexpression. ${ }^{23}$ Importantly, inhibition of heparanase activity in this model, using a TF pathway inhibitor 2 (TFPI-2)-derived peptide, was effective to attenuate the increased venous thrombus formation in aged mice and restored it to the thrombotic phenotype of younger mice. Hence, accumulation of heparanase in endothelial cells could increase the risk of age-related venous thrombosis and heparanase neutralization might be an attractive approach to attenuate the endothelial prothrombotic phenotype in this setting.

Overall, these data highlight a role of GAGs and heparanase in age-related organ senescence, which could partly explain the pulmonary and vascular pathological findings in elderly patients infected with the severe acute respiratory syndrome coronavirus 2 (SARS-CoV-2).

Environmental factors are known to be involved in the pathophysiology of respiratory and cardiovascular diseases, and may be active players in aggravating their course. ${ }^{5}$ Exposure to cadmium is known to cause damage to alveolar epithelial cells, affect their capacity to repair, and result in permanent structural alterations. HS can modulate cell responses to injury through interactions with soluble effector molecules. These interactions are often sulfate-specific, and removal of sulfate groups from HS side chains could be expected to influence cellular injury, such as that caused by exposure to cadmium. Zhang et al demonstrated that modification of HS chains by overexpression of 6-O-endosulfatase (HSulf-1), a membrane-bound enzyme which specifically removes 6-O-sulfate groups from HS side chains in two pulmonary epithelial cancer cell lines (H292 and A549) and in normal human primary alveolar type II (hAT2) cells, decreased cell viability and exacerbated the activation of apoptosis pathways. ${ }^{24}$ The cell injury was significantly intensified by cadmium. These results suggest that HS may play an important role in protection against certain environmental toxicants, such as heavy metals. Reduced levels of HS found in aged people could contribute to increased lung damage in the elderly population residing in industrial areas, particularly during the COVID-19 pandemic. A potential mechanism involved in increased disease severity in infected elderly patients is depicted in - Fig. $\mathbf{1}$.

In conclusion, increasing heparanase expression with aging results in enhanced degradation of HS chains in pulmonary tissue and blood vessels. High levels of heparanase may exacerbate immune system response and cytokine storm, thus contributing to severe lung damage. These factors may also play a role for the less favorable prognosis of aged patients

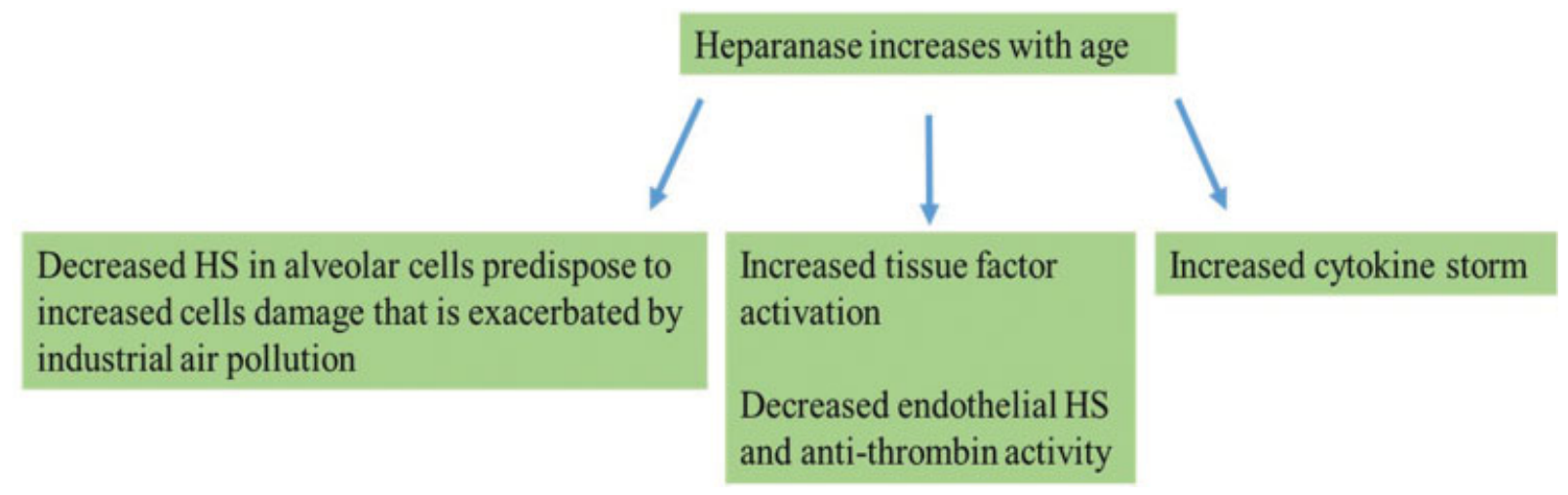

Fig. 1 Age-related heparanase effects. Elevated levels of heparanase increase cytokine levels, decrease alveolar and endothelial cell surface heparan sulfate (HS) chains, and augment tissue factor (TF) activity. These effects may predispose to enhanced lung damage and thrombosis in infections. 
infected with bacteria or viruses, including SARS-CoV-2. Thus, it may be worthwhile investigating if therapeutic modalities aiming at inhibition of heparanase effects, such as heparins or TFPI-2-derived peptides, may be capable to attenuate morbidity and mortality in infected elderly patients.

\section{Conflict of Interest}

None declared.

\section{Acknowledgment}

We would like to acknowledge the assistance of Mrs. Sonia Kamenetsky in language editing.

\section{References}

1 Martinelli I. Risk factors in venous thromboembolism. Thromb Haemost 2001;86(01):395-403

2 Tofler GH, Massaro J, Levy D, et al. Relation of the prothrombotic state to increasing age (from the Framingham Offspring Study). Am J Cardiol 2005;96(09):1280-1283

3 Mari D, Mannucci PM, Coppola R, Bottasso B, Bauer KA, Rosenberg RD. Hypercoagulability in centenarians: the paradox of successful aging. Blood 1995;85(11):3144-3149

4 Brouns SLN, Provenzale I, van Geffen JP, van der Meijden PEJ, Heemskerk JWM. Localized endothelial-based control of platelet aggregation and coagulation under flow: a proof-of-principle vessel-on-a-chip study. J Thromb Haemost 2020;18(04):931-941

5 Mannucci PM, Harari S, Martinelli I, Franchini M. Effects on health of air pollution: a narrative review. Intern Emerg Med 2015;10(06):657-662

6 Lemaitre M, Carrat F, Rey G, Miller M, Simonsen L, Viboud C. Mortality burden of the 2009 A/H1N1 influenza pandemic in France: comparison to seasonal influenza and the $\mathrm{A} / \mathrm{H} 3 \mathrm{~N} 2$ pandemic. PLoS One 2012;7(09):e45051

7 Ginaldi L, Loreto MF, Corsi MP, Modesti M, De Martinis M. Immunosenescence and infectious diseases. Microbes Infect 2001;3(10):851-857

8 Leng J, Goldstein DR. Impact of aging on viral infections. Microbes Infect 2010;12(14-15):1120-1124

9 Cambier J. Immunosenescence: a problem of lymphopoiesis, homeostasis, microenvironment, and signaling. Immunol Rev 2005;205:5-6

10 Nikolich-Zugich J. Ageing and life-long maintenance of T-cell subsets in the face of latent persistent infections. Nat Rev Immunol 2008;8(07):512-522

11 Frasca D, Riley RL, Blomberg BB. Humoral immune response and B-cell functions including immunoglobulin class switch are downregulated in aged mice and humans. Semin Immunol 2005;17(05):378-384

12 Ademokun A, Wu YC, Dunn-Walters D. The ageing B cell population: composition and function. Biogerontology 2010;11(02): 125-137

13 Liu WM, van der Zeijst BA, Boog CJ, Soethout EC. Aging and impaired immunity to influenza viruses: implications for vaccine development. Hum Vaccin 2011;7(Suppl):94-98

14 Le Saux S, Weyand CM, Goronzy JJ. Mechanisms of immunosenescence: lessons from models of accelerated immune aging. Ann N Y Acad Sci 2012;1247:69-82

15 Cavanagh MM, Weyand CM, Goronzy JJ. Chronic inflammation and aging: DNA damage tips the balance. Curr Opin Immunol 2012;24(04):488-493

16 Gutter-Kapon L, Alishekevitz D, Shaked Y, et al. Heparanase is required for activation and function of macrophages. Proc Natl Acad Sci U S A 2016;113(48):E7808-E7817

17 Digre A, Singh K, Åbrink M, et al. Overexpression of heparanase enhances $\mathrm{T}$ lymphocyte activities and intensifies the inflammatory response in a model of murine rheumatoid arthritis. Sci Rep 2017;7:46229

18 Konno K, Arai H, Motomiya M, et al. A biochemical study on glycosaminoglycans (mucopolysaccharides) in emphysematous and in aged lungs. Am Rev Respir Dis 1982;126(05): 797-801

19 Iriyama S, Matsunaga Y, Takahashi K, Matsuzaki K, Kumagai N, Amano S. Activation of heparanase by ultraviolet B irradiation leads to functional loss of basement membrane at the dermalepidermal junction in human skin. Arch Dermatol Res 2011;303 (04):253-261

20 Nitschmann E, Berry L, Bridge S, et al. Morphological and biochemical features affecting the antithrombotic properties of the aorta in adult rabbits and rabbit pups. Thromb Haemost 1998;79 (05):1034-1040

21 Nitschmann E, Berry L, Bridge S, et al. Morphologic and biochemical features affecting the antithrombotic properties of the inferior vena cava of rabbit pups and adult rabbits. Pediatr Res 1998; 43(01):62-67

22 Campisi J. Aging, tumor suppression and cancer: high wire-act!. Mech Ageing Dev 2005;126(01):51-58

23 Bochenek ML, Bauer T, Gogiraju R, et al. The endothelial tumor suppressor p53 is essential for venous thrombus formation in aged mice. Blood Adv 2018;2(11):1300-1314

24 Zhang H, Newman DR, Bonner JC, Sannes PL. Over-expression of human endosulfatase-1 exacerbates cadmium-induced injury to transformed human lung cells in vitro. Toxicol Appl Pharmacol 2012;265(01):27-42 